\title{
A Novel Rapid Conceptual Design Based on Knowledge Reuse and Evolution
}

\author{
Minghui Li, Heming Zhang, Bing Tang, Huachao Mao, Gongzhuang Peng, and Jiaxin Zhao
}

\begin{abstract}
Designers often have difficulties in fulfilling rapid conceptual design for complex products or system due to the lack of sufficient multi-disciplinary well-structured and well-evolved knowledge. Therefore, this paper presents a knowledge-based multi-disciplinary system for the conceptual design by reusing the knowledge and synthesizing the evolutionary design methodology (EDM). With the system, the designers can easily search the existing design data and successful cases for reusing or evolving the functions and parameters for the design to meet requirements.
\end{abstract}

Index Terms-Conceptual design, evolutionary design methodology, knowledge-based. multi-disciplinary system, reuse.

\section{INTRODUCTION}

Conceptual design is the earliest design stage throughout the product design. With the progress and development of technology, requirements for conceptual product design become more and more urgent, especially for designing complex products. Conceptual design stage in actual investment is only $5 \%$ of the total cost, but it affects the following design aspects and determines $70 \%$ of the total cost [1]. More seriously, as the shortening of product life cycles, users request more demands for rapid product design. Therefore, conceptual design has become more and more important in engineering product development cycle. The conceptual design starts with designing clarified requirements. It is followed by abstraction and the development of functional structure. In the end, a solution is made based on single or multiple theories [2]. Basically, the conceptual design converts the initial requirements into practical solution.

Finding ways to make the conceptual design rapid and has high-fidelity for complex products and be easy for detailed design, is the most emergent problem currently. Traditional methods [3]-[5] and software about concept design are unable to provide streamline operation, so designers cannot drastically master and use it. Another serious problem is that designers and customers can't collaborate well [5] for the product design from the traditional methods and software. Therefore our research will focus on two themes. One is the case library reusing for the rapid conceptual design. The other is the evolutionary design methodology (EDM) for the

Manuscript received November 10, 2013; revised January 15, 2014.

Minghui Li, Heming Zhang, Huachao Mao, Gongzhuang Peng, and Jiaxin Zhao are with the CIMS, Department of Automation, Tsinghua University, China (e-mail: hmz@mail.tsinghua.edu.cn).

Minghui Li and Bing Tang are with the School of Mechanical Electronic and Information Engineering, China University of Mining and Technology, China. decomposing based on the knowledge. Evolutionary design methodology $(\mathrm{EDM})$ originates from the regulation of the nature and corresponds to the rule of human cognitive competence. The process of the rapid conceptual design becomes easier for designers and customers to collaborate, which benefits from its advantages of one-sided to comprehensive and from abstract to detailed. This paper introduces EDM for rapid conceptual design and a tool to display it. With the visualized chart, the EDM is easy to be mastered and applied to research for designers. Some related works are discussed in Section II. Our evolutionary design methodology is presented in Section III. Section IV implements our approach through conceptual design software. And Section V concludes this paper.

\section{LITERATURE REVIEW}

After years of research and development, there have been many achievements for conceptual design. Through our research and study at home and abroad, there are three kinds of methods shown below.

\section{A. Conceptual Design Based on the Software Integration}

A lot of software and theories [6], [7] have been proposed for conceptual design, such as CEASIOM [8], the Computerized Environment for Aircraft Synthesis and Integrated Optimization Methods (CEASIOM) that can be used in the conceptual design process of the aircraft with emphasis on the improvement of predictive stability and the properties control achieved by higher-fidelity methods. CESAIOM offers a reliable computational method, that can replace real life model analysis during conceptual design in a fast degree. However, although most of methods can save time and simultaneously reduce product cost, these internal structures cannot be understood well by designers and unable to use in other fields.

In the aspect of computationally based engineering for conceptual design, Gregory L.Roth [9] has proposed a software named DaVinci that can enable a collaborative environment for early air vehicle systems engineering and includes a key software element that allows quickly and intuitively to build parameterized, attributed models for conceptual design to feed higher-fidelity analysis tools;

\section{B. Conceptual Design Based on the Framework}

Yong Chen et al. [10] have developed a knowledge-based framework for creative conceptual design of multi-disciplinary systems through reusing and synthesizing known principle solutions in various disciplines together; Dioclecio Moreira Camelo et al. [11] have proposed a multi-relational and interactive model for supporting the 
design process in the conceptual phase. By means of rules that connect the abstraction levels, designers can explore and converge the design space with this model; Jonathan Cagan et al. [12] have developed a framework for computational design synthesis in model and applications. The computational design synthesis is divided into these four areas: representation, generation, evaluation, guidance, which is useful in solving complex design problems. Hitoshi Komoto et al. [13] have developed a modeling framework focusing on hierarchical system decomposition and consistency management of design information to support tasks of comprehensive evaluation of descriptions from functional, parameter-level, structural, and behavioral aspects.

\section{Conceptual Design Based on the Evolution}

Parmee et al. [14] have developed an interactive evolutionary design system aiming at supporting decision-making processes during the conceptual design stages related to uncertainty, ill-definition and multiple objectives. Shih-Hsin Eve Lin et al. [15] have developed Evolutionary Energy Performance Feedback for Design (EEPFD) to support early stage design decision-making by providing rapid interaction with performance feedback. Y. Jin et al. [16] have proposed a hierarchical co-evolutionary approach to conceptual design. According to the approach, designers can decompose higher-level functions based on a set of grammar rules through a co-evolutionary computing process.

The above literature analysis discloses there are still no practical methods or software for rapid conceptual design. Therefore, the research on method of rapid conceptual design is still worth our effort.

\section{EVOLUTIONARY RAPID CONCEPTUAL DESIGN}

Evolutionary rapid conceptual design consists of two mainly parts. One is the reuse knowledge of the design knowledge for conceptual design. The other is EDM based on the design knowledge. All of them play a critical role in rapid conceptual design and are easier to be applied by designers.

\section{- The Design Knowledge}

The Design Knowledge [17] has a wide range, including product knowledge, task knowledge and process knowledge. In this paper, we divide it into the reuse knowledge and the evolution knowledge. Both contain each other, and not independent.

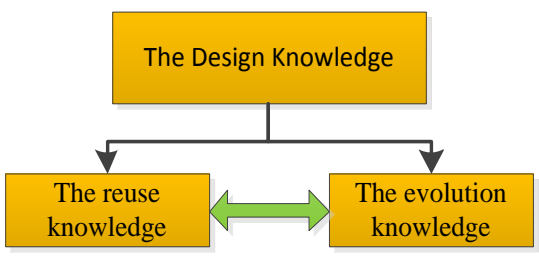

Fig. 1. Composition of the design knowledge.

\section{- The reuse knowledge}

The knowledge reuse means a method, by means of a search engine to find general formula, design parameters,
Government standards etc. to match requirements in the knowledge base.

- The evolution knowledge

The evolution knowledge represents the knowledge which can be evolved further.

- Evolutionary design methodology (EDM)

Evolutionary design methodology (EDM) is a method by means of evolving requirements and the design knowledge and reusing the design knowledge to find a solution quickly for rapid conceptual design.

\section{A. The Reuse Knowledge}

As we know, there are many researches having come up with their proposals on the conceptual design of complex products. Such as many successful complex products have been developed and are being used today. Song silyer [1] proposed that 60 percent of design activities of mechanical product in the industry are only modified a little bit, most of the rest adopt knowledge reuse. The knowledge reuse integrates in the reuse knowledge base includes mechanism knowledge base, case library and evaluation knowledge base. They play a huge role in all the process of the conceptual design, which help to save design time and improve the fidelity of conceptual design.

The mechanism knowledge base includes a lot of elements, such as general formula, empirical formula, professional knowledge, etc. Case library is equal to a data package which includes a lot of design parameters and design templates proposed by existing successful design. And evaluation knowledge base includes some government standards, ultimate parameters, economic conditions, technology conditions et al. [7]. They are illustrated in the Fig. 2. These elements are integrated together in the reuse knowledge base and can be searched when they are in use.

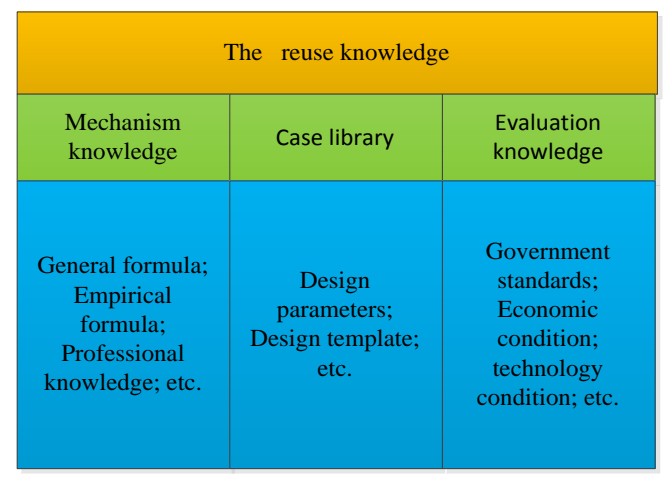

Fig. 2. The structure of the knowledge reuse.

\section{B. Evolution on the Design Knowledge}

Evolution in biology refers to genetic characters between generations. The genetic characters are the expression of gene that will be duplicated and delivered to the next generation in the process of the genetic breeding. However, gene mutation can change the expression of gene, causing genetic variation between individual organisms. The new expression will be delivered along with genes between species with species migration and horizontal gene transfer between species [18]. The theory of evolution considers that the creatures originally are evolved from non-living and the various existing creatures are evolved from common ancestors by variation, inheritance, natural selection, etc. 
According to the Evolution Theory that creatures are evolved from the non-living to the living, from the low level to high level, from the simple to complex [19], from one generation to the next generation. Similarly as the evolution of creatures, a lot of elements behind design knowledge affect its evolution. For example, there are many little parameters and formulas behind a big formula affecting its size.

Cognitive ability [20] refers to the ability of processing, storing and extracting information for the human brain and it also refers to the ability for people to master things constitution, performance and the relationship with other things, direction and driving force of things development as well as the basic rules.

In this paper, we propose an evolutionary design methodology (EDM) aiming to achieve the gradual evolution of the design process based on the knowledge for the conceptual design. The process of the evolution like biological evolution in nature is illustrated as Fig. 3. In the process of the conceptual design, at the first generation, when we face an abstract parameter (a task), and we don't know what to do and how to solve this problem, then, based on the design knowledge, we can evolve the parameter (the task) into the second generation: several specific parameters and formulas. Similarly, the second generation can be evolved into the third generation and so on. Apparently, no matter which generation they are, there are always some elements keeping the original properties and some new properties appeared like inheritance and mutation in biological evolution. As we can see from the Fig. 3, some parameters have not been evolved into the next generation, because it has been evolved into the most specific one and cannot be evolved any more or it has been evolved to the parameter that we have known like the result of the nature selection. The evolution of design process based on the knowledge makes a big abstract problem into several little specific problems, leading to a problem easier to be solved in streamline operations by designers and be consistent to the human cognitive ability.

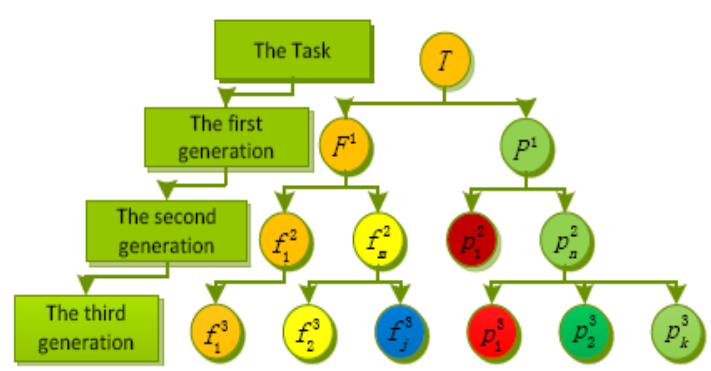

Fig. 3. Process of the knowledge evolution.

The process can be display by the following model:

$$
T_{N}^{i}=\left\langle f_{N}^{i}, p_{N}^{i}, X_{N}^{i}, Y_{N}^{i}\right\rangle
$$

$T_{N}^{i}$ represents the new task. $f_{N}^{i}$ represents functions and $p_{N}^{i}$ represent parameters of discipline in conceptual design of complex products. $X_{N}^{i}$ represent inputs, $Y_{N}^{i}$ represent outputs. $N$ represents the $N$ generation. $i$ represents the $i$ consequence of the whole $N$ generation.

$$
T_{N+1}^{i}=\left\langle f_{N+1}^{i}, p_{N+1}^{i}, \mathrm{X}_{N}^{i}, Y_{N+1}^{i}\right\rangle
$$

$f_{N+1}^{i}$ represent functions. $p_{N+1}^{i}$ represents parameters. $\mathrm{X}_{N}^{i}$ present inputs and don't change. ${ }_{N+1}^{i}$ represents outputs. $N+1$ represents the $N+1$ generation, evolved from the $N$ generation. $f$ denotes evolution of $\mathrm{F}$.

$$
f_{N}^{i}=\left\langle f_{N+1}^{i}, \mathrm{f}_{N+1}^{i+n}, \mathrm{p}_{N+1}^{i}, \mathrm{p}_{N+1}^{i+n}\right\rangle
$$

$f_{N}^{i}$ represents functions of the $\mathrm{N}$ generation, and still can be evolved into the $N+1$ generation, such as elements like

$$
\begin{aligned}
& f_{N+1}^{i}, \mathrm{f}_{N+1}^{i+n}, \mathrm{p}_{N+1}^{i}, \text { and } \mathrm{p}_{N+1}^{i+n} . \\
& \quad p_{N}^{i}=\left\langle f_{N+1}^{i}, \mathrm{f}_{N+1}^{i+m}, \mathrm{p}_{N+1}^{i}, \mathrm{p}_{N+1}^{i+m}\right\rangle
\end{aligned}
$$

$p_{N}^{i}$ represent parameters of the $\mathrm{N}$ generation, and still can be evolved into the $\mathrm{N}+1$ generation, such as elements like $f_{N+1}^{i}, \mathrm{f}_{N+1}^{i+m}, \mathrm{p}_{N+1}^{i}$, and $\mathrm{p}_{N+1}^{i+m} \cdot p$ denotes evolution of $P$.

$$
{ }_{N}^{i}=\left\langle Y_{N+1}^{i}, Y_{N+1}^{i+n}\right\rangle
$$

$p_{N}^{i}$ represent outputs of the $\mathrm{N}$ generation, and still can be evolved into the $N+1$ generation, such as elements like $Y_{N+1}^{i}$ and ${ }_{N+1}^{i+n}$.

These elements of the $N$ generation are evolved from the $N-1$ generation and can be evolved into the $N+1$ generation based on the design knowledge.

\section{Integration of the Reuse and Evolution}

The reuse and the evolution both are useful to the rapid conceptual design. The reuse can reduce design time and cost and let the mature design be applied again by using the existing parameters and methods. The evolution of parameters can be used at the part of the complex products needed to improve, making the process of design easy to be understood by designers and get the reliable conclusion for the requirements.

This paper proposes a good solution to handle the problem using the two methods that integrate those two parts. When we need to design a complex product, we can select either one or both of them meanwhile to meet requirements in the conceptual design.

The whole evolution and knowledge reuse process illustrated as Fig. 4. As we can see from the Fig. 4, when the new product s' design requirements are determined, firstly, we analyze the requirements and evolve them into several parts based the design knowledge and the practical situation. Then we select appropriate knowledge from the design knowledge base to match every part in function, structure and behavior. For example, we decide which part of the 
requirement does not need to design and which part needs to redesign or improve based on the knowledge. The parts that do not need to redesign can reuse the successful existing parameters, compositions, structure or properties which can be found in case library. The parts that need to redesign can use the general formula, professional knowledge and the evolution knowledge for the special requirements, which can be found in the mechanism knowledge base. Thirdly, by requirement match and function integration, we have several candidates, such a, b, and c. At last, we comprehensively considered about the government standards, economic conditions and technology conditions from evaluation knowledge base, choosing the optimal solution for the product design requirements. When the optimal solution is proved well to meet all the limitations of the requirement, we achieve one evolutionary design process and put it in the design knowledge base for the next requirement.

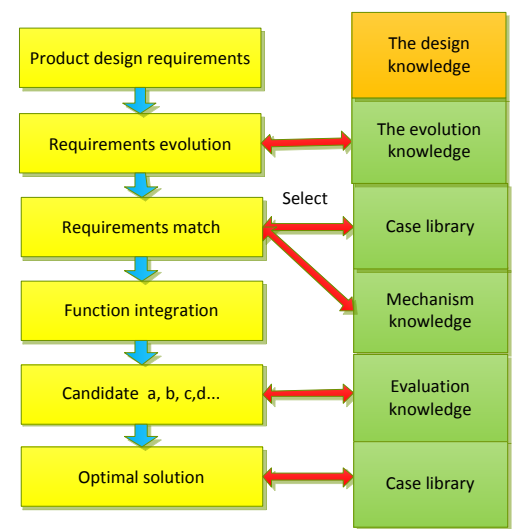

Fig. 4. Design framework based on the design knowledge.

\section{IMPLEMENTATION AND CASE STUDY}

This section shows the application of the reuse and EDM to a design case, involving a tool to display them clearly.

\section{A. Implementation}

The design case [21] begins with an initial requirement: "to improve the speed of the aircraft." With this requirement, a purpose function can be defined. Firstly, based on the EDM, we decompose the big aircraft system into several parts: the ballistic design, mass equation, power system design, shape design, structure design and control system design. Then, we analyze the composition and function of every part and decide which part does not need to redesign by using the existing data and which part need to design again by using the EDM. The whole conceptual design process is illustrated as Fig. 5.

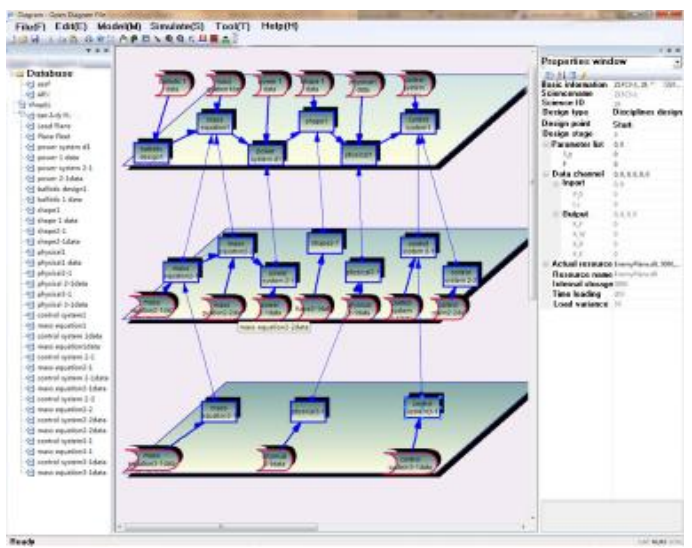

Fig. 5. The implementation of the design.

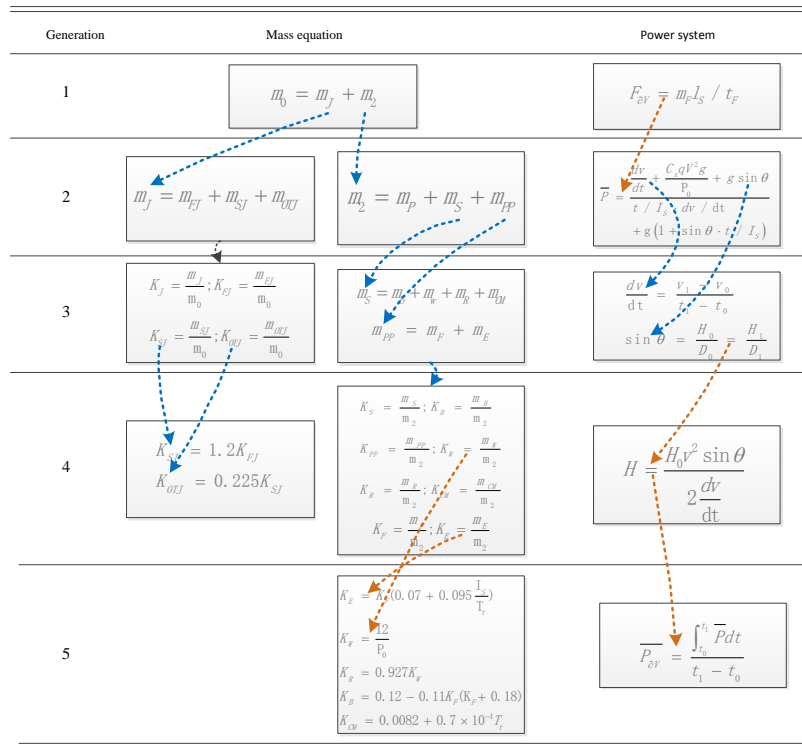

Fig. 6. Case of the aircraft design.

In the Fig. 5, region A represents the main menu where we can create a new file, edit files, model and simulate a question. Region B represents model base which includes all of the models such as data model, design model and property model. Designers can double-click on the icon on the left side of the model base and generate a corresponding model in the middle diagram. We can build relationships between the two models. Region $\mathrm{C}$ represents the property of the corresponding model, including basic information, parameter list, data channel and actual resources. We can find how many parameters that a model have and change the parameters or design stage of the model in this section.

\section{B. Case Study}

As we can see from the Fig. 5, the aircraft [18] consists of several parts. We assume that two parts of mass equation and 
power system need to redesign and others reuse the existing design parameters. Next, we evaluate the design parameters in these two parts based on EDM. The process and result displays in the Fig. 6. From the Fig. 6, we can easily understand the process of the design because the parameters are clearly evolved and from the abstract to the specific.

\section{CONCLUSIONS AND FUTURE WORK}

The proposed approach for rapid conceptual design paves a way for designers to integrate reuse and redesign into a multi-disciplinary and multi-stage system for achieving a desired function. The reuse of existing design data shorten the design cycle and reduce the waste of resources. The EDM conform to the rule of human cognitive competence and is easy to apply to other projects. Above all, designers and customers can collaborate well for the conceptual design by this approach.

Though significant progress have been made on the multi-stage system, there is still a lot of work to be done in the future, such as the case library needs to improve the search speed, the design and evaluation knowledge need to improve the fidelity of the conceptual design evolution, the extension of our approach for rapid conceptual design of multi-disciplinary and multi-stage system, et al.

\section{ACKNOWLEDGMENT}

This work is supported by The GF Basic Research Foundation of China (Grant No, A042110104) and The Natural Science Foundation of China (Grant No. NSFC61374163)

\section{REFERENCES}

[1] S. Silver et al., "Based reasoning product conceptual design system," Journal of Tsinghua University (Natural Science), pp. 6-9, 1998.

[2] Conceptual Design Study of Mechanical Products and Solutions to Solve the Problem of Process Modeling, Dalian University of Technology, pp. 131, 2008.

[3] C. Zhang et al., "A novel BOM based multi-resolution model for federated simulation," in Proc. 2013 IEEE 17th International Conference on Computer Supported Cooperative Work in Design (CSCWD) ,2013.

[4] H. Zhang, H. Mao, and G. Peng, "Modular collaborative simulation in multibody system dynamics of complex mechatronic products," in Proc. IEEE International Conference on Systems, Man, and Cybernetics, 2013.

[5] H. Mao, G. Peng, and H. Zhang, "Choosing models of appropriate resolutions for simulation: A MRM approach," AsiaSim 2013, CCIS 402, Springer, 2013, pp. 188-199.

[6] Y. Wu, "Conceptual design activities of FDS series fusion power plants in China," Fusion Engineering and Design, pp. 2713-2718, 2006.

[7] L. Wanget al., "Collaborative conceptual design- state of the art and future trends," Computer-Aided Design, vol. 34, no. 13. pp. 981-996, 2002.

[8] M. R. Afsar et al., "CEASIOM: An open source multi module conceptual aircraft design tool," International Journal of Engineering, 2013.

[9] G. L. Roth et al., "CREATE-AV DaVinci: Computationally based engineering for conceptual design," AIAA Paper, January, 2010.

[10] Y. Chen, Z. Liu, and Y. Xie, "A knowledge-based framework for creative conceptual design of multi-disciplinary systems," Computer-Aided Design, pp. 146-153, 2012.

[11] D. M. Camelo and E. Mulet, "A multi-relational and interactive model for supporting the design process in the conceptual phase," Automation in Construction, vol. 19, no. 7. pp. 964-974, 2010.

[12] M. I. Campbell, S. Finger, and T. Tomiyama, "A framework for computational design synthesis: model and applications," Journal of Computing and Information Science in Engineering, pp. 171, 2005
[13] H. Komoto and T. Tomiyama, "A framework for computer-aided conceptual design and its application to system architecting of mechatronics products," Computer-Aided Design, vol. 44, no. 10, pp. 931--946, 2012

[14] I. Parmee et al., "Introducing prototype interactive evolutionary systems for ill-defined, multi-objective design environments," Advances in Engineering Software, vol. 32, no. 6, pp. 429-441, 2001.

[15] S. H. E. Lin and D. J. G., "Designing-In performance: A framework for evolutionary energy performance feedback in early stage design," in Proc. BS2013: 13th Conference of International Building Performance Simulation Association, Chambéry, France, August 26-28.

[16] Y. Jin, W. Li, and C. Y. Lu, "A Hierarchical co-evolutionary approach to conceptual design," Annals of the CIRP, vol. 54, no. 1, pp. 155-158, 2005.

[17] D. M. Zhang et al., "A framework for design knowledge management and reuse for Product-Service," Computers in Industry, vol. 63, pp. 328 - 337, 2012.

[18] L. Shuguang, F. Y. Pei, and H. Zhimin, "Biological evolution of genetic algorithms and artificial intelligence," Dialectics of Nature, vol. 12, pp. 20-24, 1999.

[19] W. Qiuan, "Darwin's theory of evolution and natural biological evolution analysis," Hubei Social Sciences, vol. 9, pp. 90-93, 2012.

[20] M. Weeks et al., "Childhood cognitive ability and its relationship with anxiety and depression in adolescence," Journal of Affective Disorders, 2013.

[21] D. C. Brown, "Artificial intelligence for engineering design," Analysis and Manufacturing.

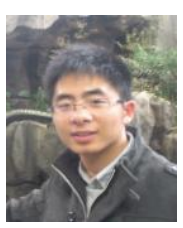

Li Minghui was born in 1988. He got his master degree from CIMS, Department of Automation, Tsinghua University, China. His research interests include modeling and simulation, the conceptual design.

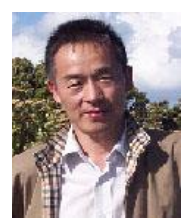

Zhang Heming was born in 1967. He is a professor and $\mathrm{PhD}$ supervisor in Tsinghua University. His research interests include multi-discipline collaborative modeling, prototype of complex product, parallel engineer and multi-resolution modeling.

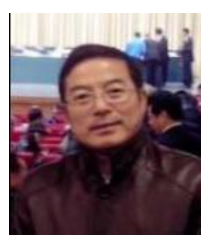

Tang Bing is an associate professor and $\mathrm{PhD}$. He is the chief librarian in China University of Ming and Technology. His research interests include coal mine safety and Mining machinery.

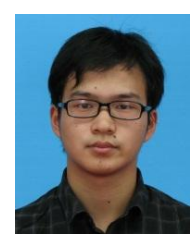

Mao Huachao was born in 1988. He got his master degree from CIMS, Department of Automation, Tsinghua University, China. His research interests include modeling and simulation, multi-resolution modeling and parallel simulation.

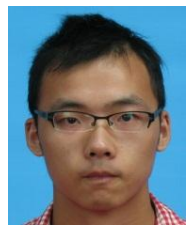

Peng Gongzhuang was born in 1990. He got his $\mathrm{PhD}$ from CIMS, Department of Automation, Tsinghua University, China. His research interests include modeling and simulation, complex system modeling, parallel simulation.

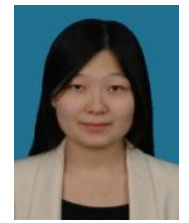

Zhao Jiaxin was born in 1990. She is pursuing her $\mathrm{PhD}$ in CIMS, Department of Automation, Tsinghua University, China. Her research interests include modeling and simulation, multi-resolution modeling and parallel simulation. 